\title{
CF GLOBAL CARE: Collaboration Between Two CF Centers; University of Michigan, USA and Marmara University, Istanbul, Turkey before and during the COVID-19 Pandemic
}

\author{
Samya Nasr ${ }^{1}$, Yasemin Gokdemir ${ }^{2}$, Ela Erdem Eralp ${ }^{3}$, Fazilet Karakoc ${ }^{3}$, Almala \\ Ergenekon ${ }^{4}$, Christopher Tapley ${ }^{5}$, Sharyn Dagher ${ }^{5}$, Sandra Bouma ${ }^{5}$, Ozge Kenis-Coskun ${ }^{6}$, \\ Damla Kocamaz ${ }^{2}$, and Bulent Karadag ${ }^{3}$ \\ ${ }^{1}$ University of Michigan \\ ${ }^{2}$ Marmara University \\ ${ }^{3}$ Marmara University Faculty of Medicine \\ ${ }^{4}$ Marmara University School of Medicine \\ ${ }^{5}$ Michigan Medicine \\ ${ }^{6}$ Dr Lufti Kirdar Kartal Egitim ve Arastirma Hastanesi
}

September 25, 2021

\begin{abstract}
Solving the world's health challenges requires multidisciplinary collaborations that bring together the talents, experiences, resources, and ideas from multiple sectors in low and middle-income countries (LMIC) and high -income countries (HIC). Cystic Fibrosis ( $\mathrm{CF}$ ) was thought to be a disease of Caucasian populations from European decent. However, it has been shown to affect people from all ethnic backgrounds. CF care varies significantly for people with CF (pw CF) from HIC with median survival approaching 50 years of age, to LMIC with pw CF dying in infancy or early childhood. To address the discrepancy in quality of care and outcomes, we report on a collaboration between our team at the University of Michigan cystic fibrosis center (UoM CFC), through support from the Middle East CF Association (MECFA) and the CF Foundation (CFF), and a CF center in Turkey (Marmara University CF Center, Istanbul) to address deficiencies and improve quality of care in that center. The collaboration has been successful in improving Ma CFC data and patient care. This partnership can be viewed as a model of collaboration to be duplicated in other Middle East Countries and LMIC to deliver optimal CF care.
\end{abstract}

CF GLOBAL CARE: Collaboration Between Two CF Centers; University of Michigan, USA and Marmara University, Istanbul, Turkey before and during the COVID-19 Pandemic

Nasr, S.Z. ${ }^{1}$

Gökdemir, Y. ${ }^{2}$

Erdem, E. ${ }^{2}$

Karakoc, F. ${ }^{2}$

Ergenekon, P. ${ }^{2}$

Tapley, C. ${ }^{1}$

Dagher, S. ${ }^{1}$

Bouma, S. ${ }^{1}$ 
Keniş Coşkun, Ö. ${ }^{2}$

Kocamaz; D.2

Karadag, B. ${ }^{2}$

${ }^{1}$ Division of Pediatric Pulmonology, Department of Pediatrics, University of Michigan, Michigan Medicine, Ann Arbor, Michigan, USA ${ }^{2}$ Division of Pediatric Pulmonology, Department. of Pediatrics Marmara University, Istanbul, Turkey

Corresponding Author: Samya Z. Nasr

Professor of Pediatrics

CF Center Director

University of Michigan, Michigan Medicine

1500 E. Medical Center DR.

Ann Arbor, Michigan, USA

Email: snasr@umich.edu

Phone: 734-764-4123

Runny Title: Collaboration Between 2 CF Centers in the USA and Turkey to Improve Care

Abstract

Solving the world's health challenges requires multidisciplinary collaborations

that bring together the talents, experiences, resources, and ideas from multiple sectors in low and middleincome countries (LMIC) and high -income countries (HIC). Cystic Fibrosis (CF) was thought to be a disease of Caucasian populations from European decent. However, it has been shown to affect people from all ethnic backgrounds. CF care varies significantly for people with $\mathrm{CF}$ ( $\mathrm{pw} \mathrm{CF}$ ) from HIC with median survival approaching 50 years of age, to LMIC with pw CF dying in infancy or early childhood. To address the discrepancy in quality of care and outcomes, we report on a collaboration between our team at the University of Michigan cystic fibrosis center (UoM CFC), through support from the Middle East CF Association (MECFA) and the $\mathrm{CF}$ Foundation (CFF), and a CF center in Turkey (Marmara University CF Center, Istanbul) to address deficiencies and improve quality of care in that center. The collaboration has been successful in improving Ma CFC data and patient care. This partnership can be viewed as a model of collaboration to be duplicated in other Middle East Countries and LMIC to deliver optimal CF care.

\section{Abbreviations:}

LMIC: Low- and middle-income countries

HIC: High income countries (HIC)

CF: Cystic Fibrosis

Pw CF: People with CF

ECFSPR: European Cystic Fibrosis Society Patient Registry

MECFA: Middle East CF Association

CFF: CF Foundation

UoM CFC: University of Michigan Cystic Fibrosis Center

MoH: Ministry of Health 


\section{Ma CFC: Marmara CF Center}

IRT: Immunoreactive trypsinogen

BMI: Body mass index

FEV1 pp: Forced Expiratory Volume at 1 second\% predicted

Kifder: Turkish CF patient and family organization

PM\&R: Physical Medicine and Rehabilitation department

IP\&C: Infection prevention and control

QI: Quality improvement methodology

CFRD: Cystic Fibrosis Related Diabetes

PT: Physical therapy

P\&PD: Percussion and postural drainage

MIC: Middle income countries

\section{Key words}

Low- and Middle- income countries, High Income Countries, Cystic Fibrosis, FEV1, BMI, QI, IP\&C

\section{Introduction}

Solving the world's health challenges requires multidisciplinary collaborations

that bring together the talents, experiences, resources, and ideas from multiple sectors in low and middleincome countries (LMIC) and high -income countries (HIC). ${ }^{1}$ In addition, a commitment to working together, can lead to the development of creative and multidisciplinary solutions required to tackle system challenges such as those in global health. ${ }^{1}$ To create an effective collaboration between institutions from LMIC and HIC, stakeholders should be willing to work together. Each stakeholder can bring varying strengths and resources to the partnerships, but they also bring their own organizational cultures, regulations, and expectations. ${ }^{1}$ Ideas to overcome geographic, economic, social, and political challenges should be addressed early in any collaboration. It is extremely important to be sensitive to cultural and religious differences in different LMIC countries. Understanding these differences helps connect people in these countries. Overcoming the cultural and language barriers in different countries/cultures can be achieved through having medical and cultural interpreters.

Cystic Fibrosis (CF) was thought to be a disease of Caucasian populations from European decent. However, it has been shown to affect people from all ethnic backgrounds. ${ }^{2} \mathrm{CF}$ care varies significantly for people with $\mathrm{CF}$ (pw CF) from HIC with median survival approaching 50 years of age, ${ }^{3}$ to LMIC with pw CF dying in infancy or early childhood. LMIC face significant challenges to promote CF diagnosis and improvements in $\mathrm{CF}$ care due to financial constraints and significant burden of other diseases. ${ }^{4}$ Health disparities for pw $\mathrm{CF}$ in LMIC can result from limited access to clinical care owing to lack of its availability on a national or local level, to limited diagnostic tools for the disease in some countries, to restriction of access to specialized services or therapies (financial or physical), and/or to lack of knowledge or education regarding how the disease should be treated, resulting in poor outcomes. ${ }^{5}$ Western European countries show significantly different clinical outcomes compared to countries in Eastern Europe including Turkey as shown from the European Cystic Fibrosis Society Patient Registry (ECFSPR). ${ }^{6}$ Disparities could be due to several factors including differences in resources and available medications. A paucity of resources and medication contributes to suboptimal treatment, lower lung function, poor nutrition, poor quality of life and shortened lifespan.

To address the discrepancy in quality of care and outcomes, we report on a collaboration between our team at the University of Michigan cystic fibrosis center (UoM CFC), through support from the Middle East CF 
Association (MECFA) and the CF Foundation (CFF), and a CF center in Turkey (Marmara University CF Center, Istanbul) to address deficiencies and improve quality of care in that center.

\section{Background}

In Turkey, there are approximately 3,000 pw CF. CF centers are supported by the Turkish Ministry of Health $(\mathrm{MoH})$. CF clinical outcomes in Turkey have not followed those reported in the USA and Western European Countries. CF in Turkey shows a disproportionate rate of morbidity and mortality, as indicated by the current number of CF patients at Marmara CF Center (Ma CFC) with most patients are below 18 years of age (297 patients are 0-17 years of age and only 70 patients are 18 years and above in 2020). Most patients with CF in Turkey are children and their quality of life is poor. They are likely to have low body mass index (BMI) and low forced expiratory volume at 1 second \% predicted (FEV1\% predicted (FEV1pp)). Ma CFC is the largest in Istanbul and second largest in Turkey. The center has infrastructure support including a CF nurse, Physical therapist, and a part time dietitian, sweat testing lab, genetic testing, and microbiology laboratory. However, the allied health support was inadequate for the size of that center.

A newborn screening program for CF started in 2015 which uses immunoreactive trypsinogen (IRT) combined with repeat IRT, followed by sweat testing, and DNA sequencing. Sweat testing laboratories are using the coulometric method, and it was certified by $\mathrm{MoH}$. This method was found to be compatible with classic sweat testing methods using Gibson \& Cooke and Chloridometer Methods. ${ }^{7}$ F508del homozygous mutations is present in only $28 \%$ of the patients. The most common mutations are F508del, 1677delTA, N1303K. Ma CFC has been reporting to the ECFSPR since 2017. Median age at diagnosis and follow-up duration of the patients was 0.3 (IQR:0.2-0.9) and 11.9 (IQR:5.4-16.1) years, respectively. Most CF medications are available in Turkey, covered by the government and free for patients. There is a Turkish CF patient and family organization (KIFDER). This organization has been very active in lobbying on behalf of pw CF and $\mathrm{CF}$ centers with the government and, also is very involved in CF education, in collaboration with CF centers across Turkey.

UoM CFC follows approximately 275 pediatric patients and over 600 total pw CF. The center data has been above average for USA centers and is well supported with adequate staffing. The center is very active in research and quality improvement (QI) work.

\section{Deficiencies}

The collaboration started with a site visit in 2018. The following deficiencies were identified:

1. A high number of patients had low BMI Z scores: Median Z score was -1.2 (25th and 75th: -2.2- -0.2 ), -0.2 (25th and 75th: -1.5-0.4), and -0.6 (25th and 75th: -1.3-0.1) in patients 2-5, 6-11, $12-17$ years old, respectively. The BMI Z score was lower in all age groups compared to the peers registered in ECFSPR.

2. A high number of patients had FEV1 pp values which trended down as they got older: Median FEV1 pp was 86.1 ( $25^{\text {th }}$ and $75^{\text {th } \%: 70.5-97.8) ~ i n ~ 6-17 ~ y e a r s ~ c o m p a r e d ~ t o ~} 60.8$ (25th and 75 th: $\left.40.5-73.3\right)$ in patients 18-24 years old.

3. Inadequate structural integration of Ma CFC with Physiotherapy and Physical Medicine and Rehabilitation department (PM\&R). Patients were receiving consultation for airway clearance, bronchial hygiene techniques and exercise programs as needed especially while hospitalized.

4. The center had no infection prevention and control (IP\&C) policies or measures in place.

5. Although there was an outpatient clinic schedule in place, patients with mild to moderate CF exacerbation were seen in the CF clinic without a scheduled appointment.

6. Clinic space was inadequate and not suitable for CF patients follow up.

7. Inadequate Allied Health professionals support to the center, including CF nurse, dietetian, physical therapist. Ma CFC had no social workers or psychologists in the institution..

\section{Focus of the Collaborations}

1) Train and engage the Ma CFC team in quality improvement methodology (QI) to help address the clinical deficiencies. 
2) Improve clinical outcomes for pw $\mathrm{CF}$ at Ma CFC as measured by BMI Z score and FEV1 pp., using quality improvement methods used by UoM CFC. ${ }^{8,9}$

3) Engage and educate pw CF as partners in their care through collaboration with KIFDER.

4) Achieve improvement in median life expectancy and quality of life using evidence-based, state of the art healthcare delivery, operating under strict QI principles. Also, continue ongoing multidisciplinary team training, and establishing a meaningful clinical research initiative.

\section{Methods}

Following the site visit, in 2018, the collaboration started in 2019 and included exchange of visits between the 2 centers to share knowledge and experience and help train the Ma CFC team on the CFF standard of care. The collaboration included in person visit from the UoM CFC team to Ma CFC, visit from the Ma CFC team to the UoM CFC and a rotation for the Ma CFC fellow to train at the UoM CFC for 2 months. In 2020 and 2021, due to the CoV2 pandemic and the travel restrictions, webcast meetings were arranged and are ongoing between the 2 centers to continue with follow up on progress and QI efforts. QI methodology was taught to all team members to improve their center data including patients' nutritional and pulmonary function status. Implementation of IP\&C policy started following the first site visit to the center. UoM CFC QI processes were shared with Ma CFC team members. Ma CFC has been involved in several clinical research projects. Expanding Ma CFC's research capacity is a focus of the collaboration as well. The CFF guidelines were reviewed and adjusted to meet the Ma CFC's needs and available resources. The Patient/Family organization KIFDER involvement with this collaboration has been very important to help change the current culture of pw CF. KIFDER has been helpful in getting pw CF to adhere to the new implemented IP\&C measures, not to socially interact and to schedule regular visits for exacerbations. In addition, KIFDER has been crucial in supporting the center with much needed resources and staff support through fund raising and advocacy. $\mathrm{MoH}$ is supportive in supplying most of the necessary medications, diagnostics and laboratory follow up. However, it has been partially supportive of the centers' Allied Health professionals. Table 1 summarizes the Ma CFC patient population demographics.

\section{Results}

After the initial meeting with the hospital administration, two more examination rooms were allocated to the center. A strict IP\&C policy was initiated with the support of the Hospital Infection Control Committee and administration. The required personal protective equipment like gowns, masks, and gloves were provided in both the outpatient clinic and the inpatient. A second CF nurse was added; both nurses are funded by donations raised by KIFDER. The fellow's visit to UoM CFC was a good opportunity to see the multidisciplinary approach to CF care. She observed the importance of the teamwork in the follow-up of pw CF and recognized the importance of strict infection control.

To address the decreased pulmonary funtion for pw CF, UoM CFC standardized patient respiratory care protocols and algorithms were implemented at Ma CFC. The focus of the QI project was CF patients 6-18 years old with FEV1 pp $<80$, however, all protocols and algorithms were also applied to all patients in the center. The QI project was initiated in June, 2019, and ended on 10/2020. Flow charts from each member of the care team were created, standardized CF care algorithm was implemented and an individualized treatment plan for each patient was created to address barriers to adherence to the treatment plan that may result in FEV1 pp <80. Baseline, 6th and 12th month mean FEV1\%pred was $62.2 \pm 15.1,67.2 \pm 17.7$, $70.3 \pm 19.0$ respectively. Overall, there was significant improvement in mean FEV1 pp by $7.3 \%$ in 6 months $(\mathrm{p}<0.001)$ and by $10.1 \%$ in 12 months $(\mathrm{p}<0.001) .{ }^{10}$ The algorithms and protocols became part of the center follow up. Table 2 shows the comparison of FEV1pp values of 2017 vs. 2020 according to age groups

The hospital administration increased the dietary support to 2 full time dietitians. Through the collaboration, the dietitians learned additional evaluation tools for a more in-depth assessment of patients' nutritional status, including pediatric malnutrition criteria. In addition, a QI project was started to help improve the nutritional status of pw $\mathrm{CF}$ at Ma CFC. Growth and development were evaluated according to the protocol 
determined within the scope of the QI project. While height and weight were evaluated prior to the project, BMI was added to the evaluation. Recommendations for increasing caloric intake were made as indicated after the evaluation of the food consumption records of the patients. Oral nutritional supplements were recommended as needed. Pancreatic enzyme replacement therapy was maximized. In addition, nutrition education about the importance of maximizing the caloric intake was given to $\mathrm{pw} \mathrm{CF}$ and their families.

Appetite stimulants started to be used for children in the higher risk group as defined by the QI project protocol. When enteral nutrition is indicated, pw CF and their families were informed in advance and the plan was discussed in detail to gain their agreement. Appropriate nutritional supplements were started through the G-tube, and appropriate enzyme dosing was ensured. Summary of the initial work was presented in North American CF Conference. ${ }^{11}$ Table 3 shows Comparison of BMI Z-score values in 2017 vs. 2020 according to the age groups

To address the poor adherence to airway clearance techniques, patients' low activity levels, and poor percussion and postural drainage, the 2 teams worked together to improve patient information materials, including developing booklets for patient education that also included section on improving hygiene and nebulizer cleaning and disinfection. In addition, the physical therapy team (PT) team at Ma CFC has implemented a one-on-one teaching to improve patient adherence and a regular evaluation of percussion and postural drainage $(\mathrm{P} \& \mathrm{PD})$ and aerobic exercises regularly was started. Patients' positive expiratory devices are screened in every visit, and their High Frequency Chest Wall Oscillation Devices (VEST), if available are screened yearly for proper use. The PT team also visits the patients during hospitalizations for follow up during acute exacerbations and for implementation of P\&PD and aerobic exercises. Also, hearing, and vestibular dysfunction symptoms and bowel and bladder incontinence symptoms have been added to the evaluation during regularly scheduled clinic visits.

A focus on improving the knowledge of CF caregivers was done through standardized training programs supported by various educational material. To improve nebulized machines cleaning and disinfection, a halfday education program was started once a week for 10-12 caregivers every week. One hundred and ninety caregivers received this education till the start of the COVID-19 pandemic. Pre and post-tests were completed by the caregivers and the results of this project was published in Journal of Pediatric Pulmonology. ${ }^{12}$ New education material regarding infection control and aerosol treatment were developed and shared with pw CF and their care givers.

Annual depression and anxiety screening of pw $\mathrm{CF}$ and their caregivers was started via standardized questionnaires. Results of 2 years of screening were published in Pediatric Pulmonology. ${ }^{13}$ Patients with high level of depression and/or anxiety levels were referred to the child psychiatry department for evaluation and treatment. Depression and anxiety were found to be common in pw CF and their families. As a result of the $\mathrm{CoV} 2$ pandemic, three virtual resources were developed and shared with pw $\mathrm{CF}$ and their families: breathing exercises for children 3-12 years old, group virtual therapy for adolescent, and support group for the parents of children 0-3 years old.

\section{Discussion}

Our team (UoM CFC) started collaborating with a CF center in Turkey (Ma CFC) to see if we can help improve their CF care. Our collaboration has been successful because the two teams have been willing to work together to create an effective model of collaboration. Each team brought varying strengths and resources to the partnerships, but they also brought their own organizational cultures, regulations, and expectations, that led to adjustment of the guidelines and protocols to accommodate for the cultural, regulations and resources. Marmara University administration were also willing to work with the teams to improve care which led to providing the center with much needed resources.

The goal of the collaboration has been to address the deficiency in quality of care and outcomes. The collaboration between the 2 centers has been supported by MECFA and CFF. Of note, Turkey is classified as middle-income country. 
To create a model of care at Ma CFC, UoM CFC team visits to the center was an important part of the collaboration to evaluate the areas that need improvement and follow progress. The Ma team visits to the UoM CFC provided an important look at a HIC CF center's structure and operation. The collaboration included also training of the Ma team on QI measures and processes.

Data sharing between the two centers was an important part of the collaboration.

Teaching and implementing QI tools have led to improvement in the Ma CFC care and data. Compared to 2017, there has been significant improvement in FEV1 in all age groups in 2020 data (Table 2). This is due in large part to the close monitoring of FEV1pp and the intervention if there was a drop in FEV1pp according to UoM algorithm. ${ }^{9}$ Improvements in FEV1pp are also likely due to teaching pw CF and their families the importance of doing their treatment regularly, adhere to the regularly scheduled visits and learn how to clean their nebulized equipment. In addition, educational material was created and shared with them through classes and virtually. Evaluating the nutritional status of pw $\mathrm{CF}$ has become a top priority since achieving an optimal nutritional status results in improving morbidity and mortally. The UoM protocols and algorithm were used for the implementation of a BMI QI project at Marmara. ${ }^{8}$ BMI improved for the 2-5-year-old age group but it didn't show improvement in the 6-11 years old and the 12-17- year -old groups. That could be due to the need to more aggressive in implementation of the algorithm in these age groups including the use of high calorie diet, appetite stimulants and enteral feedings. That could also be due to the need to change the culture of pw $\mathrm{CF}$ and families of accepting the fact that these patients are small and want to be thin. (Table 3 ).

The collaboration started with in person interactions. The site visit, team visits and fellow's visits have all been very productive and useful to both teams. However, when the CoV2 pandemic started, an adjustment to the collaboration was needed. The communications became virtual with regularly scheduled virtual meetings to work on progress, start new projects and come up with new ideas. The second fellow's rotation was delayed and rescheduled to 2022. The collaboration was scheduled for 2 years, however with the pandemic delay, it became 2 years in person visits (with the second year scheduled for 2022), and two years of virtual interaction in between

. The extension of the collaboration has been fruitful in strengthening the collaboration and the QI work.

Next steps in the collaboration are: 1- to work with the Turkish Thoracic Society and MoH to create a notional CF guideline, 2- to create National CF Center Network in Turkey, using evidence-based, state of the art healthcare delivery, operating under quality improvement principles, ongoing multidisciplinary team training, and establishing a meaningful clinical research initiative.

In conclusion, this partnership can be viewed as a model of collaboration to be duplicated in other Middle East Countries and LMIC to deliver optimal CF care. There are future challenges and opportunities to create a meaningful collaboration between teams in LMIC and HIC to improve CF care globally. Some of the challenges include the inability to make the diagnosis, either because of lack of the sweat testing equipment or low index of suspension for CF. Another challenge could be the lack of the multidisciplinary team training and approach to treating CF. Opportunities include collaborations with CF teams from HIC to help with the training and to help adapt CF care guidelines to the countries' resources and culture. Creating patient organizations would help in increasing awareness like KIFDER does in Turkey. Use of mobile technology (which is widely available in most LMIC), to strengthen the collaboration between teams and to facilitate access, improve communication between the medical teams and patients and families would be beneficial. Creating registries with standard datasets that guide population management and data management tools could help clinical management. We believe that more collaborations between CF centers in HIC and LMIC could lead to significant improvement in CF care worldwide.

\section{References}

1. Taylor, RM and Alper, J; National Academies of Sciences, Engineering, and Medicine 2018. Exploring Partnership Governance in Global Health: Proceedings of a Workshop. Washington, DC: The National 
Academies Press. Pages 1-4. https://doi.org/10.17226/25069.

2. Bell SC, Mall MA, Gutierrez H, Macek M, Madge S, Davies JC, et al. The future of cystic fibrosis care: a global perspective. Lancet Respir Med 2020;8(1):65-124.

3. Cystic Fibrosis Foundation (2020) 2019 patient registry annual data report. https://www.cff.org/Research/Researcher-Resources/ Patient-Registry/2020-Patient-RegistryAnnual-Data-Report.pdf.

4. Ferreira da Silva Filho, LVR, Zampoli M, Malena Cohen-Cymberknoh, M, Kabra, SK; Cystic fibrosis in low and middle-income countries (LMIC): A view from four different regions of the world. In press, Paediatric Respiratory Reviews. https://doi.org/10.1016/j.prrv.2020.07.004.

5. Kerem E, Cohen-Cymberknoh M. Disparities in cystic fibrosis care and outcome: socioeconomic status and beyond. Chest 2016;149(2):298-300.

6. European Cystic Fibrosis Society. ECFS patient registry. http://www. ecfs.eu/projects/ecfs-patientregistry. Accessed October 1, 2019.

7. Gokdemir, Y., Vatansever, P., Karadag, B., Seyrekel, T., Baykan, O., Ikızoglu, N.B., Ersu, R., Karakoc, F., and Haklar, G.: Performance Evaluation of a New Coulometric Endpoint Method in Sweat Testing and Its Comparison with Classic Gibson \& Cooke and Chloridometer Methods in Cystic Fibrosis. Clinical Trial article, Front. Pediatr., 2018 May 22 May;6:133. https://doi.org/10.3389/fped.2018.00133.

8. Ramírez, I., Filbrun, A., Hasan, A., Kidwell, K., Nasr, S.Z.: "Improving Nutritional Status in a Cystic Fibrosis Center." Pediatric Pulmonology, 2015; 50:544-551.

9. Filbrun, A.G., Enochs, C., Caverly, L., Rajala, K., Powell, C., Merrick, E., Nasr, S.Z.:" Quality Improvement Initiative to Improve Pulmonary Function in Pediatric Cystic Fibrosis Patients." Pediatric Pulmonology. 2021; 56:1527-1533. https://DOI:10.1002/ppul.25017.

10. Gokdemir, Y, Erdem Eralp, E, Ergenekon, P, Yilmaz Yegit, C, Mursalığlu, H, Uzunoğlu, B, Kocamaz, D, Taştan, G, Filbrun, A, Enochs, C, Bouma, S, Iwanicki, C, Karakoc, F, Nasr, S.Z., Karadag, B.: Change Iin FEV1 after Iimplementation of Standardiized Cf Care Algoriithm-Quality Improvement Project. Presented in a poster presentatin at the Virtual NACFC September 29- October 2, 2021.

11. Gokdemir, Y., Erdem Eralp, E., Ergenekon, P., Yilmaz Yegit, C., Mursalığlu, H., Uzunoğlu, B., Kocamaz, D, Taştan, G., Filbrun, A., Enochs, C., Bouma, S., Iwanicki, C., Karakoc, F., Nasr, S.Z., Karadag, B.: "Change in Body Mass Index of Children with CF After Implementation of Standardized Nutritional Algorithm-Quality Improvement Project." Presented in a poster session at the Virtual North American CF Conference (NACF). October 20- October 24, 2020.

12. Yilmaz Yegit, C., Ergenekon, P., Mursaloglu, H., Cenk, M., Suzer Uzunoglu, B., Tastan, G., Gokdemir, Y., Erdem Eralp, E., Duman, N., Karahasan Yagci, A., Karakoc, F., Nasr, S., Karadag, B.; "The Effects of Nebulizer Hygiene Training on the Practices of Cystic Fibrosis Patients Caregivers." Pediatric Pulmonology. Published on line February 16, 2021. doi: 10.1002/ppul.25307. [Epub ahead of print] PubMed PMID: 33538406.

13. Mursaloğlu, H.H., Yegit, C.Y., Ergenekon, A., Gökdemir, Y., Erdem Eralp, E., Karakoç, F., Nasr, S.Z., Karadăg, B.; "Screening of Depression and Anxiety in Cystic Fibrosis Patients/ Caregivers and Evaluation of Risk Factors." Pediatric Pulmonology. Published on line Jan. 29, 2021. doi: 10.1002/ppul.25295. [Epub ahead of print] PubMed PMID: 33512091.

\section{Hosted file}

MaMi Tables.docx available at https://authorea.com/users/436731/articles/538848-cf-globalcare-collaboration-between-two-cf-centers-university-of-michigan-usa-and-marmarauniversity-istanbul-turkey-before-and-during-the-covid-19-pandemic 